\title{
A clinical enigma of ongoing constrictive pericarditis
}

\author{
Authors: Deacon Zhao Jun Lee, ${ }^{A}$ Reshma Amin, ${ }^{B}$ John Baksi ${ }^{C}$ and Robert Gerber ${ }^{D}$
}

\begin{abstract}
A 59-year-old lady presented with a 1-week history of orthopnoea, paroxysmal nocturnal dyspnoea, night sweats and a productive cough. She had no recent history of travel. Transthoracic echocardiogram revealed preserved left ventricular systolic function with abnormal pericardial thickening and restrictive left ventricular filling consistent with pericardial constriction. Cardiac magnetic resonance imaging confirmed a globally thickened pericardium and showed evidence of pericardial inflammation and constrictive physiology. She did not respond to diuresis, pulsed intravenous steroids or broad spectrum antibiotics and multiple investigations were negative, including autoimmune screen and tuberculosis cultures. Eventually a serum sample was found to be positive for Strongyloides stercoralis and it emerged that this lady had travelled to Egypt 8 years previously, where it is thought that she contracted $S$ stercoralis leading to her developing constrictive pericarditis. This case report summarises the presentation and progression of this case and rare diagnosis.
\end{abstract}

KEYWORDS: Cardiology, pericardial disease, pericarditis, Strongyloides stercoralis

\section{Background}

Patients with symptoms consistent with congestive cardiac failure are commonly encountered on the acute medical take. Constrictive pericarditis is not often encountered as the underlying cause and in this case report we present a rare occurrence of Strongyloides stercoralis leading to constrictive pericarditis in a patient potentially infected 8 years prior to presentation.

\section{Case presentation}

A 59-year-old lady presented with a 1-week history of orthopnoea, paroxysmal nocturnal dyspnoea, night sweats and productive cough. Prior to presentation she was living

Author: ${ }^{\text {A }}$ specialty registrar, East Sussex Healthcare NHS Trust, St Leonards-on-sea, UK; ${ }^{B}$ senior house officer, East Sussex Healthcare NHS Trust, St Leonards-on-sea, UK; ' ${ }^{\text {C }}$ Consultant cardiologist, Royal Brompton and Harefield NHS Foundation Trust, London, UK;

${ }^{D}$ consultant cardiologist, East Sussex Healthcare NHS Trust, St Leonards-on-sea, UK independently. Her past medical history included chronic obstructive pulmonary disease, Barretts' oesophagus, chronic back pain and depression. She was not on long-term steroid therapy and she did not report any history of recent foreign travel.

On initial examination, she had oxygen saturations of $95 \%$ on air, a heart rate of $82 \mathrm{bpm}$ and blood pressure of 122/78 $\mathrm{mmHg}$. Jugular venous pressure was elevated and apex beat was undisplaced. Bibasal crepitations were audible on auscultation. Non-tender pulsatile hepatomegaly was palpable.

Following extensive investigation, it was clear that the patient had evidence of constrictive pericarditis and she had several hospital re-admissions in the ensuing months. There was a real diagnostic dilemma as no organisms were found on prolonged microbiology testing. Eventually, pulsed intravenous methylprednisolone was started on the suspicion that the cause of her presentation was inflammatory in origin. Unfortunately this led to significant clinical deterioration 2 days later with respiratory failure, which was presumed to be florid chest sepsis triggered by steroid administration and this required further broad spectrum antibiotic therapy. Eventually, serological testing using ELISA (enzyme-linked immunosorbent assay) was positive for $S$ stercoralis and ivermectin therapy was commenced, leading to significant clinical improvement.

\section{Investigations}

An electrocardiogram showed global T wave inversion and initial blood tests revealed a normocytic anaemia. Renal biochemistry was normal but alkaline phosphatase was raised at $192 \mathrm{IU} / \mathrm{L}$. Inflammatory markers on initial presentation were elevated with a C-reactive protein of $115 \mathrm{mg} / \mathrm{L}$ and erythrocyte sedimentation rate of $59 \mathrm{~mm} /$ hour. White blood cell count was $9.2 \times 10^{9} / \mathrm{L}$ with neutrophils of $6.0 \times 10^{9} / \mathrm{L}$ and eosiniphils of $0.1 \times 10^{9} / \mathrm{L}$.

Chest X-ray revealed a moderate right-sided effusion. This was confirmed on computerised tomography imaging of the chest, which also demonstrated mild mediastinal lymphadenopathy, likely reactive.

Ultrasound of the abdomen was unremarkable.

Transthoracic echocardiography revealed abnormal pericardial thickening and restrictive left ventricular filling consistent with pericardial constriction (Figs 1 and 2, Video S1 and S2). On Doppler assessment, mitral inflow variation appeared greater than $25 \%$. Left ventricular systolic function was preserved with an ejection fraction of $53 \%$. 


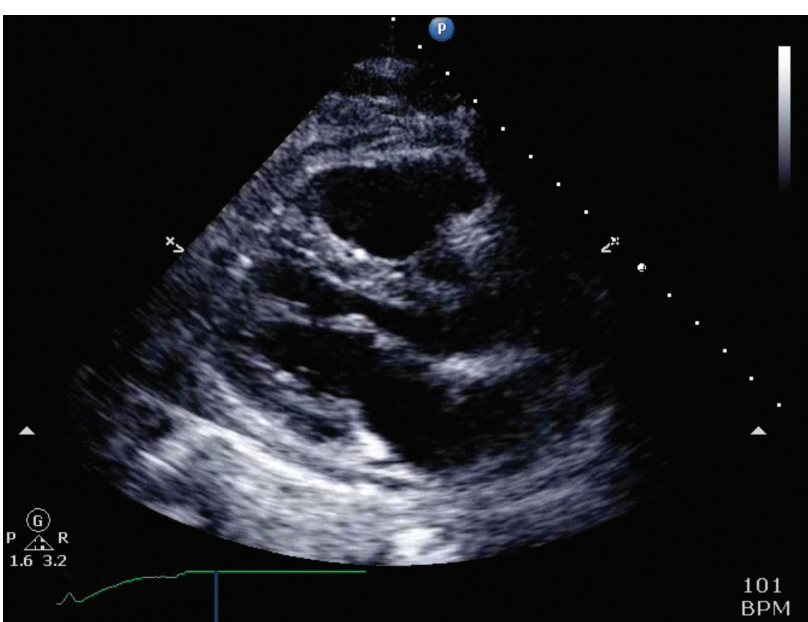

Fig 1. Echocardiogram showing parasternal long axis. See supplementary file (Video S1) for video demonstrating abnormal pericardial filling and restrictive left ventricular filling.

Cardiac magnetic resonance imaging showed globally thickened pericardium predominantly around the right ventricle. There was evidence of pericardial inflammation and the images were consistent with constrictive physiology (Fig 3, Video S3).

Endobronchial ultrasound and transbronchial needle aspiration, which were performed because of the mediastinal lymphadenopathy, were negative.

Several other causes of her presentation were excluded during her subsequent hospital admissions, including lymphoma, HIV and autoimmune disease. No organisms were found in either pleural or pericardial fluid, including tuberculosis.

Left and right heart catheterisation was performed and showed subcritical disease in the left main stem and left anterior descending artery. There was elevation and equalisation of end-diastolic pressures in keeping with pericardial constriction.

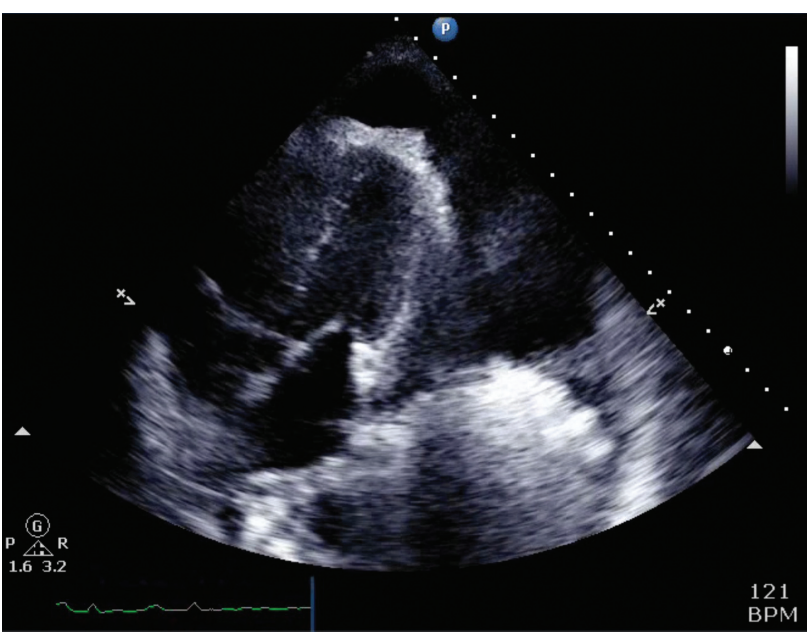

Fig 2. Echocardiogram showing apical view. See supplementary file (Video S2) for video showing septal bounce consistent with pericardial constriction.

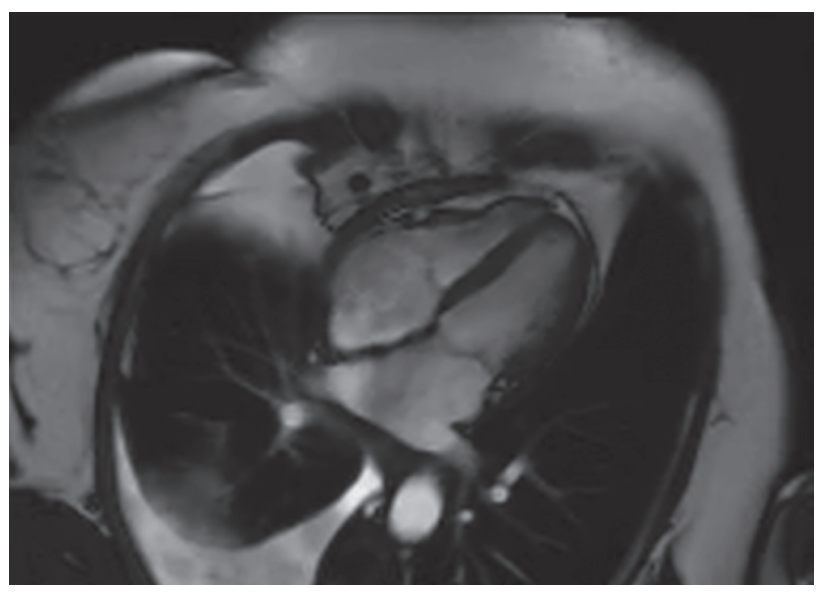

Fig 3. Cardiac magnetic resonance image. See supplementary file (Video S3) for video showing evidence of pericardial inflammation and constrictive physiology.

\section{Outcome and follow-up}

At this stage, surgical treatment options, including pericardiectomy and coronary artery bypass grafting, were discussed with the cardiothoracic surgeons, but the risks of cardiac surgery were felt to be too high. She was eventually discharged from hospital and required long-term oxygen therapy, but otherwise made a satisfactory recovery. Constrictive physiology was still present on repeat transthoracic echocardiography in the ensuing months and this was managed conservatively as she was unfit for surgery.

\section{Discussion}

Constrictive pericarditis is an uncommon disease that often mimics other pathologies. There is fibrous thickening of the pericardium, which impedes normal diastolic filling of the heart. This leads to a reduction in stroke volume by reducing venous return. This commonly results in symptoms of exertional dyspnoea, fatigue and can cause an elevated jugular vein pressure.

The majority of causes in the western world are idiopathic or occur post-surgery. The most common cause worldwide is tuberculosis.

The pathophysiological hallmark of pericardial constriction is equalisation of end-diastolic pressures on cardiac catheterisation, which occurs because filling is determined by limited pericardial volume and not the compliance of the chambers themselves. ${ }^{1}$

The gold standard treatment is pericardiectomy, which results in rapid symptomatic and haemodynamic relief. ${ }^{2,3}$

There are few documented cases of $S$ stercoralis associated with constrictive pericarditis. $S$ stercoralis is an intestinal nematode that most commonly results in chronic infection of the gastrointestinal tract via the skin and lungs. Symptoms include diarrhoea, abdominal discomfort, nausea and anorexia. It is usually diagnosed by detecting larvae in stool samples. Eosinophilia in infected patients is common. ${ }^{4}$

$S$ stercoralis is found mainly in tropical and subtropical regions - such as Africa, Brazil and parts of Central America 
and South-East Asia. An estimated 30-100 million people are infected worldwide although in many endemic countries, information on infection rates is limited. In developing countries, it is more prevalent in rural areas where sanitary conditions are inadequate. ${ }^{5}$

In hyperinfection syndrome due to $S$ stercoralis, the larvae lifecycle accelerates and increases the parasite burden. This leads to disseminated disease, defined as disease outside the gastrointestinal tract, lungs or skin. Hyperinfection syndrome is more common in immunocompromised patients, transplant patients and patients with HIV or haematological malignancies.

Ivermectin has been found to be the most efficacious drug in treating disseminated $S$ stercoralis infection. ${ }^{6}$

\section{Learning points}

$>S$ stercoralis is a rare but important cause of constrictive pericarditis. Taking a travel history is an essential part of the patient assessment.

$>$ Hyperinfection syndrome or disseminated disease in $S$ stercoralis can follow administration of steroids.

$>$ Unless treated, $S$ stercoralis can cause lifelong infection; therefore, patients can become infected and present clinically many years later.

\section{Supplementary material}

Supplementary videos may be found in the online version of this article at www.clinmed.rcpjournal.org/:

S1 - Parasternal long axis view demonstrating abnormal pericardial filling and restrictive left ventricular filling.

S2 - Apical view showing septal bounce consistent with pericardial constriction.

S3 - Cardiac magnetic resonance imaging evidence of pericardial inflammation and constrictive physiology.

\section{Conflicts of interest}

The authors have no conflicts of interest to declare.

\section{Author contributions}

DZJL contributed to the drafting and editing of the manuscript. RA contributed to the drafting and editing of the manuscript. JB contributed to the diagnosis of the patient's underlying condition and editing of the manuscript. RG contributed to the diagnosis of the patient's underlying condition and editing of the manuscript.

\section{Acknowledgements}

We would like to thank Dr Zoe Ottaway (senior house officer, Conquest Hospital) and Dr Osei Kankam (consultant respiratory physician, Conquest Hospital) for their involvement with the patient's care.

Consent to publish the clinical details and images in the article was given by the patient's next of kin after she subsequently passed away.

\section{References}

1 Little WC, Freeman GL. Contemporary reviews in cardiovascular medicine: pericardial disease. Circulation 2006;113:1622-32.

2 Schwefer M, Aschenbach R, Heidemann J, Mey C, Lapp H. Constrictive pericarditis, still a diagnostic challenge: comprehensive review of clinical management. Eur J Cardiothoracic Surg 2009;36:502-10

3 Ling LH, Oh JK, Schaff HV et al. Constrictive pericarditis in the modern era: evolving clinical spectrum and impact on outcome after pericardiectomy. Circulation 1999;100:1380-6.

4 Ericsson CD, Steffen R, Siddiqui AA, Berk SL. Diagnosis of Strongyloides stercoralis infection. Clin Infect Dis 2001;33:1040-7.

5 Schar F, Trostdorf U, Giardina F et al. Strongyloides stercoralis: global distribution and risk factors. PLoS Negl Trop Dis 2013;7:e2288

6 Kassalik M, Mönkemüller K. Strongyloides stercoralis hyperinfection syndrome and disseminated disease. Gastroenterol Hepatol 2011;7:766-8.

Address for correspondence: Dr Deacon Zhao Jun Lee, Conquest Hospital, The Ridge, St Leonards-on-Sea, East Sussex TN37 7RD, UK

Email: deacon.lee.04@aberdeen.ac.uk 